\title{
La teoría del decrecimiento y el proceso de enseñanza-aprendizaje de lenguas
}

\section{Degrowth Theory and the Language Teaching-Learning Process}

\author{
Luis Guillermo Barrantes-Montero ${ }^{1}$ \\ Universidad Nacional \\ Escuela de Literatura y Ciencias del Lenguaje \\ Heredia, Costa Rica \\ luisba66@yahoo.es
}

Recibido 14 de marzo de 2014 • Corregido 30 de julio de 2014 • Aceptado 22 de agosto de 2014

\begin{abstract}
Resumen. Esta disertación se propone mostrar cómo las consecutivas crisis que enfrentan las sociedades regidas por la economía de mercado involucran todas las esferas de generación y difusión del conocimiento y demandan de ellas serias redefiniciones, tanto en sus presupuestos epistemológicos como en sus acciones sustantivas. Más exactamente, se analizan los procesos de enseñanza-aprendizaje de lenguas a la luz de la teoría del decrecimiento, como un planteamiento alternativo a las tendencias de instrumentalizar las lenguas a favor de la legitimación de la vorágine de producción y consumo que amenaza el futuro global.
\end{abstract}

Palabras claves. Decrecimiento, desarrollo, economía, lingüística aplicada, enseñanza del inglés.

Abstract. This paper aims to show how the consecutive crises faced by those societies ruled by a market economy pervade all areas of generation and transfer of knowledge. Such crises also demand serious redefinitions from societies in both their epistemological assumptions and their substantive actions. More precisely, the processes of language teaching and learning are viewed in the light of the Degrowth Theory, as an alternative approach to the trends of using the teaching of languages as a means of legitimizing the production-consumption vortex that threatens the global future.

Keywords. Degrowth theory, development, economics, applied linguistics, English teaching.

\footnotetext{
${ }^{1}$ Magíster en la Enseñanza del Inglés de la Universidad de Costa Rica. Licenciado en Traducción Inglés-Español de la Universidad Nacional, Costa Rica. Licenciado en Teología, Universidad Francisco Marroquín, Guatemala. Bachiller en Filosofía de la Universidad Francisco Marroquín, Guatemala. Docente del área de inglés de la Universidad Nacional, Costa Rica.
} 
doi: http://dx.doi.org/10.15359/ree.18-3.15

URL: http://www.una.ac.cr/educare

CORREO: educare@una.cr

La época de la modernidad y, sobre todo, la época de la explosión tecnológica ha difundido por doquier los conceptos de desarrollo y crecimiento como la gran tarea de la humanidad. En palabras de Carmen (2004), el desarrollo viene a ser "la religión sustituta de la segunda mitad del siglo XX" (p. 15). En consecuencia, cada sociedad ligada al mundo occidental ha debido tomar las medidas necesarias para contemporizar con el derrotero marcado por el progreso y la tecnología.

No obstante, la riqueza mundial creció de manera desigual hasta el extremo de que la sobreabundancia de bienes y servicios ha traído consigo el deterioro masivo de la naturaleza y un sinfín de nuevos males en la salud y calidad de vida de la gente de los países centrales. Por su parte, en las naciones periféricas el desarrollo siempre fue "maldesarrollo" (Carmen, 2004, p. 33) y los problemas humanos y ambientales son mucho mayores que en el mundo desarrollado. Ante la evidencia, reconocida por unos y ocultada por otros (George, 2000) de que ese orden económico no mejora la vida de la gente del planeta ni la garantiza a largo plazo, ha ido emergiendo la idea de que el mundo debe entrar en un proceso de desaceleración del crecimiento descontrolado. En eso consiste la teoría del decrecimiento (Taibo, 2009).

En este trabajo se pretende establecer un puente entre esa teoría y el papel decisivo que desempeña la enseñanza de lenguas, sobre todo del inglés. La difusión de ese idioma puede contribuir positivamente a la formación de conciencia en la población aprendiente acerca de la necesidad de corregir el camino del maldesarrollo. Como también puede ayudar a perpetuar la engañosa idea de que para las naciones del Tercer Mundo no parece haber más alternativa que emular a las del Primero y que la clave para lograrlo es aprendiendo sus lenguas. Se espera contribuir a crear un talante crítico entre docentes, investigadores y diseñadores de políticas educativas.

\section{La teoría del decrecimiento ante la vorágine producción-consumo en el mundo occidental}

Al abordar el tema del binomio producción-consumo en los países de corte occidental -entiéndase tanto los comprendidos dentro del llamado primer mundo, como aquellos en vías de desarrollo y cuyas economías dependen directamente de los primeros- conviene iniciar con una referencia al término globalización desde la perspectiva de sus críticos. Por ejemplo, según Latouche (2003), este concepto solo es un modo más de designar la mundialización de los mercados; o bien, la mercantilización del mundo, su economización.

Por su parte, la genial obra de George (2000), El informe Lugano, plantea las estrategias que los mismos defensores del capitalismo neoliberal sugieren a sus tomadores de decisiones para corregir los yerros, los déficits y las contradicciones que la implementación de este orden económico ha traído consigo. En una de las reflexiones-recomendaciones del informe se destaca lo siguiente: 
doi: http://dx.doi.org/10.15359/ree.18-3.15

URL: http://www.una.ac.cr/educare

CORREO: educare@una.cr

La economía está en el centro de la sociedad, pero los efectos sociales indeseables podrían ser lo suficientemente fuertes como para debilitar los beneficios económicos. Las empresas deben seguir teniendo libertad para invertir y prosperar siempre que las condiciones sean idóneas, pero las personas que dejan atrás se comportarán de una forma impredecible y desestabilizadora. Hay que evitar la regulación excesiva, pero un mercado totalmente desregulado (o autorregulado) corre el riesgo de ir hacia la autodestrucción porque, abandonado a sí mismo, generará demasiado pocos ganadores y demasiados perdedores, más excluidos que incluidos. (p. 36)

Ante el panorama anterior, se presenta ahora, aunque de forma somera, la teoría que apuesta por un decrecimiento en los distintos países. De acuerdo con esta visión, urge reducir la producción y el consumo "porque vivimos por encima de nuestras posibilidades, porque [hay que] ... cortar emisiones que dañan peligrosamente el medio y porque empiezan a faltar materias primas vitales" (Taibo, 2009, p. 71).

Un ejemplo paradójico de ello lo constituye la extracción de un mineral llamado coltán, el cual es usado en la industria electrónica por sus altas propiedades como conductor de electricidad, sobre todo en teléfonos móviles y otros aparatos tecnológicos (Tettay, 2012). El control de ese insumo industrial ha provocado guerras fratricidas en los países donde se extrae, sobre todo en el Congo, así como explotación y maltrato de los obreros, en especial los niños. Ahora que los teléfonos celulares se convierten en un artículo casi de primera necesidad en muchas sociedades de todo el planeta, al usuario consumidor solo le interesa el producto final, sus características y su precio; para nada le inquieta el rastro de sangre y de destrucción que queda en el proceso. Lo mismo ocurre con una interminable lista de otros artículos que se usan y consumen cotidianamente.

El decrecimiento sostiene que las generaciones presentes no deben seguir una línea indefinida de crecimiento y abuso del entorno natural a expensas de lo que les ocurre a las comunidades locales, en especial campesinas e indígenas y lo que les ocurrirá a las generaciones futuras de todos los estratos sociales. Para Latouche (2003), el desarrollismo ha logrado permear la totalidad las esferas del imaginario. Cuando la propaganda comercial y el discurso demagógico ponen de moda una idea y la difunden masivamente, la población llega a creer que lo que las cosas que se publicitan con tanta insistencia y con el auspicio de las autoridades es porque debe ser lo mejor. Entonces, si se quiere visualizar algún atisbo de cambio, se debe trabajar con ahínco en el análisis del discurso de las llamadas "palabras tóxicas". Entre estas, según Gómez y Saxe (2010) cabe mencionar conceptos como "desarrollo sostenible", gobernabilidad", "cambio climático", "flexibilidad laboral","reforma", entre otros. Latouche (2003) se refiere a esos conceptos así:

La descolonización del imaginario pasa por la crítica de los conceptos, incluyendo el de las "palabras habituales", cuando los hombres y las mujeres que las utilizan son víctimas a su vez de manipulación. El desarrollo y la economía son el problema y no la solución. (p.147) 
doi: http://dx.doi.org/10.15359/ree.18-3.15

URL: http://www.una.ac.cr/educare

CORREO: educare@una.cr

Esas palabras tóxicas son consideradas tales porque conllevan significados de un sesgo manipulador que pretende orientar a la opinión pública hacia la creencia de que no hay nada de malo en las prácticas habituales de la maquinaria industrial y comercial. Solo por mencionar un ejemplo: el concepto de "cambio climático" (Saxe, 2006) es un eufemismo que esconde y mitiga las reacciones que podría tener entre la gente el caer en la cuenta de que las variaciones en el clima son consecuencias del impacto humano en la naturaleza mucho más que de procesos naturales a los que no se les debe temer.

La cita de Gómez y Saxe (2010) nos pone en perspectiva para darle sentido al título de este trabajo. La enseñanza de lenguas y el modo de producción neoliberal capitalista mantienen una relación muy estrecha y poderosa: gracias al potencial que la enseñanza de lenguas, sobre todo del inglés, posee como medio para influir en el modelamiento de la cosmovisión de los estudiantes, la industria del libro, de los materiales didácticos y de otras actividades lucrativas asociadas les presentan a los aprendientes el ideal de soñar con un mundo concebido a la medida de sus intereses, incuestionable, el único posible. Cook (2003) advierte de ello en los siguientes términos:

... the role of English as a global lingua franca, arises doubts about the association in many EFL materials of the English language with specific cultural practices, usually those of the dominant mainstream culture in either Britain or the USA. [... el papel que juega el inglés como lengua franca global hace surgir dudas acerca de la relación existente entre muchos materiales para la enseñanza del inglés con prácticas culturales específicas, en particular, las de la corriente cultural dominante, ya sea en su versión inglesa o estadounidense]. (p. 58)

Los docentes, permeados de esa visión tecnócrata de las cosas, ayunos quizás de una formación epistemológica que les permitiera entender su profesión de manera holística y como parte de un proyecto de nación a largo plazo y no como un inmediatismo irresponsable, devienen un instrumento óptimo para los fines del gran capital. De manera consciente o no contribuyen a la difusión de opciones lingüísticas, las cuales, de acuerdo con Cook (2003), construyen valores y significados.

\section{El ajuste de la producción del conocimiento a las demandas económico-empresariales: EI caso de la enseñanza del inglés y otras lenguas}

En el apartado anterior se confrontó la tendencia desarrollista del orden económico imperante con la teoría del decrecimiento. Corresponde ahora presentar la manera en que los presupuestos teóricos de ese orden se llevan a la práctica mediante las directrices que emanan de las instituciones financieras internacionales. Tales directrices adquieren un carácter bastante superior al de meras "recomendaciones" cuando se giran a las naciones periféricas, altamente endeudadas ante el grupo Banco Mundial (BM) y organismos regionales asociados. 
doi: http://dx.doi.org/10.15359/ree.18-3.15

URL: http://www.una.ac.cr/educare

CORREO: educare@una.cr

Los estudios que se llevan a cabo con el auspicio del BM e incluso de organizaciones ideológicamente afines como la UNESCO, en el caso de la educación, plantean medidas conducentes a mejorar lo que, desde su perspectiva, se entiende por calidad y eficiencia, en esas economías. Así, por la vía de los tratados de libre comercio, se les conmina a entrar en competencia en condiciones que no podrían ser más desiguales. Además, se violenta incluso el derecho de autodeterminación de los pueblos, la idiosincrasia cultural, la seguridad alimentaria y hasta la misma soberanía nacional, como consecuencia de los lineamientos de los planes de ajuste estructural.

No cabe duda de que la educación constituye un pilar fundamental en el sistema de producción capitalista. Sin embargo, el capitalismo neoliberal, calificado por algunos de neoconservadurismo (Dale y Robertson, 2004), concibe la educación como un activo más del sistema productivo, un bien transable y susceptible a ser convertido en objeto mercantil.

A diferencia de las aproximaciones epistemológicas humanistas que reconocen en la educación un derecho inherente al ser humano mediante el cual este puede cultivar su propio perfeccionamiento de manera holística, la "lógica" del mercantilismo globalizador ve en las instituciones educativas más bien instancias o nichos de formación del capital humano al servicio de la industria tecnológica y del comercio. Los individuos, no ya las personas, en vez de formados, son entrenados o adiestrados para desempeñar un oficio. Su estabilidad laboral, la cual es un sustrato indispensable de su equilibrio bio-psico-social (Alpi y Quiceno, 2006), es decir, de su salud, queda a merced de la contingencia y volatilidad de las demandas del mercado.

La enseñanza del inglés, tal y como se promueve hoy, está orientada a coincidir con esas demandas mercantilistas. Cuanto más se invierte en ella, más parece agudizarse la desigualdad y los conflictos sociales que esta conlleva. Por esa razón, urge una trasformación de paradigmas en el propósito de la enseñanza de lenguas. No se comprende cómo los docentes, los diseñadores de planes de estudio y los directores de programas de enseñanza de lenguas continúen creyendo que el enfoque epistemológico actual de la enseñanza de lenguas no tiene alternativa. Entre las mismas organizaciones que apoyan financieramente la enseñanza del inglés en países del tercer mundo se emiten informes que critican severamente el modo en que se toman las decisiones. Un informe de la Fundación Ford acerca de los proyectos de ayuda en el campo de la educación (Phillipson, 2003, citando a Fox, 1975), dice así:

...the Western models that served as the basis for developmental assistance in pre-university education were, in fact, not adequate. At their best, at the university level they were minimally transferable. At other levels, attempts to apply such models to developing societies often created more problems than were solved. What was missing was detailed knowledge among American and British aid agencies of how the educational system worked in the countries they were attempting to assist and the language setting that surrounded those systems. [... los modelos occidentales que sirvieron de base para la ayuda al desarrollo en la educación pre-universitaria fueron, de hecho, inadecuados. A lo sumo fueron escasamente 
doi: http://dx.doi.org/10.15359/ree.18-3.15

URL: http://www.una.ac.cr/educare

CORREO: educare@una.cr

transferibles a nivel universitario. En los demás niveles, los intentos de aplicar tales modelos a las sociedades en desarrollo con frecuencia crearon más problemas de los que resolvieron. Lo que faltó fue un conocimiento detallado, por parte de las agencias de ayuda estadounidenses y británicas de cómo funcionan los sistemas educativos de los países que intentaban ayudar y del trasfondo de lenguaje que rodea esos sistemas]. (p. 12)

Es posible trascender esa cita y afirmar que lo inadecuado de los modelos occidentales de ayuda y la falta de conocimiento de los sistemas educativos de los países en desarrollo por parte de sus contrapartes cooperadoras no fueron, ni siguen siendo, los únicos impedimentos para que tales contribuciones dieran resultado. Es necesario, además, reconocer que no ha habido un verdadero interés por parte de las autoridades políticas ni por el sector académico de los países beneficiarios por analizar los verdaderos propósitos de esas ayudas ni por adecuarlas a la realidad social, económica y cultural de sus pueblos.

Al no existir criticidad, ni siquiera para administrar las ayudas, se ha condescendido con el despliegue de un estilo de vida -y de consumo- $ß$ en la población muy poco acorde con sus posibilidades reales. El Tercer Mundo se ha vuelto tan desarrollista e irresponsable en sus hábitos de consumo, como el Primero. Y la enseñanza del inglés, en buena medida, ha contribuido con ello. Por tal razón, como lo afirma Phillipson (2003), "seems to be essential for those concerned with the teaching and learning of Englishtoquestion thelanguae pedagogy profesionalism we hae inherited. [parece esencial que quienes nos preocupamos por la enseñanza y el aprendizaje del inglés cuestionemos el profesionalismo en la pedagogía de lenguaje que hemos heredado]" (p. 13).

\section{Hacia una redefinición de los procesos de enseñanza-aprendizaje de lenguas desde la perspectiva del decrecimiento}

Las naciones periféricas sufren un nuevo colonialismo a todo nivel y a ello contribuye la enseñanza de lenguas, pues la propuesta temática del currículo y de los materiales didácticos persuade al estudiantado de que el aprendizaje de la lengua extranjera implica, de modo concomitante, la adopción de las pautas de comportamiento de las sociedades cuya lengua se aprende. Muchos talleres de capacitación de docentes de inglés enseñan maneras de hacer las clases más "atractivas", "lúdicas" y"tecnológicas" $y$, de un modo sutil e implícito, de ilusionar a los estudiantes con la ficción de que al consumir productos y cambiar hábitos de vida, de alguna manera se van a acercar a las sociedades anglohablantes de países desarrollados.

En vista de lo anterior, resulta apremiante que la formación de docentes de lenguas incluya dos componentes esenciales. El primero consiste en conferirle a la enseñanza y aprendizaje de la lengua extranjera una dimensión realista en cuanto a las expectativas que tal emprendimiento puede generar. Como profesionales, el personal docente debe conocer el ámbito deontológico de su disciplina. Deben resistirse, por ejemplo, a proponer el aprendizaje de la lengua como la opción ideal para alcanzar altas metas laborales, sin enseñar también a 
sopesar las posibilidades concretas que ese aprendizaje le abre a cada aprendiente en relación con las limitaciones que pueda tener cada cual. Eso se logra mediante el entrenamiento de docentes en el desarrollo del pensamiento crítico, que deberá estar presente en cada lección que prepare e imparta.

El segundo se refiere a la contextualización del acto de aprendizaje en concordancia con la realidad inmediata de los aprendientes (Tudor, 2000) y la del mundo como tal. Desde la perspectiva del decrecimiento, la enseñanza de lenguas, lejos de continuar alimentando la vorágine del consumo desplegada mediante los libros de texto y las actitudes acríticas de los docentes debería, más bien, aprovechar el espacio y el momento para el cultivo de la interculturalidad, del reconocimiento de la diversidad y del fortalecimiento de las propias identidades. Ello, de alguna manera, contribuiría a detener el afán que envuelve a la gente de hoy por poseer cuanto le presenta la propaganda comercial, aunque para ello deba vivir continuamente endeudada.

Un ejemplo claro del modo que se puede enseñar un idioma con sensibilidad de decrecimiento puede ser la siguiente: En una determinada clase de inglés con habilidades integradas, al igual que en cada unidad, corresponde diseñar una lección de comprensión de lectura. El docente, que planea su lección con sentido crítico, puede sustituir la lectura que presenta el libro de texto por otra que proponga un contenido más acorde con su propósito. $\mathrm{O}$ bien, si dispone de tiempo suficiente, puede incluir una lectura complementaria. Las etapas de pre-lectura, durante la lectura y post-lectura constituyen el espacio de que dispone el docente para desarrollar actividades que ayuden a sensibilizar a los estudiantes, más que dar gratuito asentimiento a lo que proponen los libros de texto.

La enseñanza de un idioma, por el contacto intercultural que permite, es un espacio privilegiado para incentivar la reflexión entre los aprendientes, entre otras cosas, de que el paso de las personas por este mundo implica una responsabilidad con los demás y con el planeta, que las metas profesionales no deben centrarse en adquirir un aumento en la capacidad de consumo y que la plenitud humana no se alcanza con tener más sino con ser más. Como mínimo, esa reflexión debería enseñar a las personas a racionalizar el gasto.

La contribución a un talante de decrecimiento es posible, entonces, también en el aprendizaje de lenguas. No es un secreto que el llamado"progreso" de determinadas sociedades tiene lugar en relación directa con el sacrificio de las fuerzas, los recursos y el entorno de otras. Un desempeño profesional, en el sentido estricto de la palabra, por parte de los docentes de lenguas, los desarrolladores de currículos y los productores de materiales didácticos, supone que no intenten abstraerse de esa ineludible constatación.

Por lo anterior se puede afirmar que, con un compromiso serio por el futuro de la gente y del planeta, es posible introducir, en medio de esa inmediatez, la idea de que todo aprendizaje es parte de un proyecto integral de vida. Que la educación y la profesionalización del ser humano deben estar imbuidas de una opción por racionalizar el gasto y propiciar el ahorro. Además que, frente a la competitividad que promueve el mercado neoliberal, es posible creer 
doi: http://dx.doi.org/10.15359/ree.18-3.15

URL: http://www.una.ac.cr/educare

CORREO: educare@una.cr

en la complementariedad, en el bien común. En otras palabras, es preciso que la formación de profesionales de lenguas modernas realice un giro epistemológico hacia una perspectiva humanista.

\section{La importancia de los planteamientos alternativos en la epistemología y en la acción sustantiva de las disciplinas científicas}

En principio, la academia debe ser independiente. Al esbozar su fundamento epistemológico, las disciplinas científicas necesitan contar con total soltura de compromisos institucionales y de intereses particulares. Ciertamente, la razón de ser de cada una de ellas consiste en su grado de incidencia en las preocupaciones del ser humano. Pero no todas las preocupaciones humanas son de naturaleza material, ni toda actividad de producción intelectual es mensurable en términos económicos.

La filosofía de la ciencia reflexiona no tanto sobre el qué cuanto sobre el cómo se realiza el quehacer científico. Del modo en que se dilucide esa interrogante derivará el actuar en concreto de la ciencia, su acción sustantiva. El neo-conservadurismo de la globalización hegemónica de hoy busca mantener el status quo. Sin embargo, los profesionales de las distintas disciplinas científicas deben tener presente que hay modos alternativos de hacer ciencia, epistemologías alternativas. Santos (2011), por ejemplo, propone una epistemología del Sur:

Entiendo por epistemología del Sur el reclamo de nuevos procesos de producción y de valoración de conocimientos válidos, científicos y no científicos, y de nuevas relaciones entre diferentes tipos de conocimiento, a partir de las prácticas de las clases y grupos sociales que han sufrido de manera sistemática las injustas desigualdades y las discriminaciones causadas por el capitalismo y el colonialismo. (p. 35)

Lo que ha ocurrido en el orden económico que domina el mundo de hoy es que la puesta en práctica del conocimiento que produce la academia ha logrado una acumulación exponencial de recursos materiales e intelectuales para determinados actores sociales y, en consecuencia, de su poder de decisión. Este último se entiende tanto en el ámbito de las relaciones privadas entre individuos como en el de las relaciones internacionales y globales.

En tal contexto, ha acontecido una especie de shock epistemológico en muchos centros de educación superior: se ha dado una invasión del campo de competencias del objeto material de la ciencia sobre su objeto formal. Por eso ahora es la práctica, la producción, la acción sustantiva -y sustantivada-, la que pretende dictarle a la filosofía cómo debe discurrir acerca de la ciencia. Una colonización de la filosofía. Esto ha acontecido, en buena parte, por lo que Narváez (2010) concibe como: “La pretensión gubernamental de participar en el mercado global del conocimiento aplicando en el campo de la educación y la cultura criterios modernos anglosajones como la mercantilización de la educación" (p. 266). 
En el campo específico de las ciencias de la educación, muchos currículos están diseñados para que los docentes ejecuten planes de estudios en franca consonancia con los intereses de los grupos de poder. La justificación es bastante contundente: ellos les pagan sus salarios. En este escenario, la ciencia de la educación está renunciando a ser tal y ha aceptado el modesto papel de gestora y ejecutora de directrices. Parecería que ya la teoría de la educación no hace falta, lo que cuenta es disponer de nuevos sujetos de producción y consumo. Lo que en otros tiempos se conocía como investigación académica ha ido cediendo terreno al cumplimiento de los requisitos que exigen los programas de acreditación y evaluación de calidad de las carreras universitarias.

Ante esa realidad, la enseñanza de lenguas puede decantarse alternativamente como una vía para educar y concienciar a la gente acerca de la necesidad de frenar el despilfarro de los recursos y de revertir la mentalidad de indolencia ante la explotación humana. Es posible que comiencen a irrumpir en la historia generaciones de gente que anteponga el ser al poseer, enmienden el error del camino desarrollista y rescaten la humanidad.

Como se indicó en el apartado anterior, aprender una lengua extranjera puede y debe ser una experiencia vital, que trascienda el mero hecho de adquirir habilidades de comunicación con gentes de otras latitudes. Supone, más bien, una expansión del espectro de humanización de la persona, al tener mayor conciencia de la otredad, de la diversidad, a la vez que un creciente entusiasmo por hacer del bienestar integral una experiencia colectiva.

\section{Conclusión}

Se ha intentado exponer en esta disertación el nexo existente entre la teoría del decrecimiento, que emerge ante la irrefrenable ambición materialista de controlar el mundo y poseer sus recursos que subyace en la mentalidad de los grupos de poder económico y político de los países ricos, y la enseñanza de lenguas, sobre todo del idioma inglés. Este, debido a su posicionamiento como lengua universal, funciona de hecho como un instrumento para adoctrinar a la población en la dinámica producción-consumo, pero también puede ser un instrumento muy valioso para dar a conocer modos alternativos de vida en el planeta que fomenten la inclusión de las personas; que hagan disminuir las prácticas de explotación; que fomenten el compartir los recursos, sobre todo el de la información, y que garanticen el entorno natural para las generaciones que están por venir.

Ante lo que se ha denominado en este trabajo como la vorágine de producción-consumo, se presentó la teoría del decrecimiento, según la conciben autores como Cook (2003), Gómez y Saxe (2010) y Latouche (2003). Tales fuentes coinciden en que la capacidad del planeta para satisfacer las demandas de consumo, que para las grandes mayorías de la población mundial apenas busca satisfacer necesidades básicas, mientras para las minorías privilegiadas se incluyen además hábitos de consumo suntuoso e irresponsable, ya asoma atisbos de colapso global.

Se ha hecho referencia a la necesidad de una transformación paradigmática en la mentalidad de la gente, lo cual solo puede lograrse mediante la educación. Se presentó la enseñanza y el aprendizaje de lenguas como locus privilegiado para contribuir en ese sentido. 
doi: http://dx.doi.org/10.15359/ree.18-3.15

URL: http://www.una.ac.cr/educare

CORREO: educare@una.cr

De manera específica, se ha hecho énfasis en la necesidad de formar docentes de idiomas en el pensamiento crítico. Ellos pueden ser agentes catalizadores del mensaje impulsor del consumo que subyace en los libros de texto. En cada lección, el profesorado puede implementar actividades de contrapeso a la propuesta desarrollista de los textos y de la propaganda comercial, para que sus estudiantes procuren distinguir lo que es provechoso y conveniente para sí, de lo que no lo es en el contacto con las culturas extranjeras. Se puso como ejemplo el caso de las actividades comprensión de lectura en los cursos de inglés con habilidades integradas. Al definir los objetivos para las etapas de pre, durante y post lectura, es posible suscitar ideas conducentes a la sensibilización social, al refrenamiento de la actitud consumista y a la racionalización del uso de los recursos. Lo mismo se puede alcanzar en las demás habilidades de comprensión y producción. De ese modo, la enseñanza de lenguas realizará grandes aportes a la transformación de la mentalidad ciudadana, con el fin de formar generaciones que luchen con responsabilidad, sensatez y sentido común por sociedades conscientes, inclusivas y protectoras del entorno.

El tema que se ha abordado es polémico y su estudio se encuentra más bien en ciernes, por lo que la revisión de bibliografía al respecto es todavía limitada. No obstante, la apuesta por el decrecimiento ya ha comenzado a ganar simpatizantes en todo el mundo. Por ello, se ha considerado conveniente y oportuno establecer un puente entre la enseñanza de lenguas y esa nueva visión de la convivencia humana y de las relaciones de producción-consumo entre las sociedades. El personal docente, con una formación holística y muy responsable, podrá contribuir a darle un giro más humano a esas relaciones.

Queda, para estudios posteriores, la publicación de estudios de casos y experiencias de docentes que deseen compartir los resultados de su puesta en práctica de esteenfoque pedagógico.

\section{Referencias}

Alpi, S. V., Sierra y Quiceno, J. M. (julio-diciembre, 2006). Salud y enfermedad desde el modelo mágico al bio-psico-social de la psicología de la salud. El Ágora USB, 6(2), 165174. Recuperado de http://web.usbmed.edu.co/usbmed/elagora/docs/agora10/ Agora\%20Diez\%20(Tema\%201\%20Salud\%20y\%20Enfermedad\%20desde\%20el\%20 modelo\%20magico).pdf

Carmen, R. (2004). Desarrollo autónomo. Humanizar el paisaje: Una incursión en el pensamiento y la práctica radicales. Heredia, Costa Rica: EUNA.

Cook, G. (2003). Applied Linguistics [Lingüística aplicada]. Oxford: Oxford University Press. doi: http://dx.doi.org/10.1093/applin/24.2.249

Dale, R. y Robertson, S. (2004). Interview with Boaventura de Sousa Santos. [Entrevista con Boaventura De Sousa Santos]. Globalisation, Societies and Education, 2(2), 147-160. doi: http://dx.doi.org/10.1080/14767720410001733629

George, S. (2000). Informe Lugano. Barcelona: Icaria editorial. 
doi: http://dx.doi.org/10.15359/ree.18-3.15

URL: http://www.una.ac.cr/educare

CORREO: educare@una.cr

Gómez, J. y Saxe, E. (marzo, 2010). El Banco Mundial y la educación superior comercializada. La universidad pública comercializada: Caso de la Universidad Nacional de Costa Rica (UNA). Ponencia presentada en el Congreso Internacional de Educación Superior. Universidad Nacional, Facultad de Filosofía y Letras. Recuperado de http://www.una.ac.cr/bibliografia_/ components/com_booklibrary/ebooks/CB_CIESUP_07.pdf

Latouche, S. (2003). Decrecimiento y post desarrollo. El pensamiento creativo contra la crisis del absurdo. Madrid: El viejo Topo.

Narváez, A. (julio-diciembre, 2010). Educación, capitalismo y desarrollo. Cultura alfabética y globalización anglosajona. Signo y Pensamiento, 29(57), 248-267. Recuperado de http:// www.redalyc.org/pdf/860/86020052016.pdf

Phillipson, R. (2003). Linguistic Imperialism [Imperialismo lingüístico]. Oxford: Oxford University Press.

Santos, B. (julio-septiembre, 2011). Epistemologías del Sur. Utopía y Praxis Latinoamericana, 16(54), 17-39. Recuperado de http://dialnet.unirioja.es/servlet/articulo?codigo=4231309

Saxe, E. E. (2006). Colapso mundial y guerra (2a ed.). San José, Costa Rica: Amo al Sur Editorial. Recuperado de http://es.scribd.com/doc/145244805/33135782-Eduardo-E-SaxeFernandez-Colapso-Mundial-y-Guerra

Taibo, C. (2009). En defensa del decrecimiento. Sobre el capitalismo, crisis y barbarie. Madrid: Los libros de la Catarata.

Tettay, J. C. (3 de diciembre de 2012). El coltán. C. el Colombiano. Recuperado de http://www. elcolombiano.com/BancoConocimiento/E/el coltan/el coltan.asp

Tudor, I. (2000). The Dynamics of the Language Classroom [La dinámica de la clase de lenguas]. Cambridge: Cambridge University Press.

\section{Cómo citar este artículo en APA:}

Barrantes-Montero, L. G. (setiembre-diciembre, 2014). La teoría del decrecimiento y el proceso de enseñanza-aprendizaje de lenguas. Revista Electrónica Educare, 18(3), 251261. doi: http://dx.doi.org/10.15359/ree.18-3.15

Nota: Para citar este artículo en otros sistemas puede consultar el hipervínculo "Como citar el artículo" en la barra derecha de nuestro sitio web: http://www.revistas.una.ac.cr/index.php/EDUCARE/index 